\title{
Prospective multicentre validation study of a new standardised version of the 400- point hand assessment
}

\author{
Michel Konzelmann ${ }^{1,2^{*}}$ (D), Cyrille Burrus ${ }^{1,2}$, Colette Gable ${ }^{3}$, François Luthi ${ }^{1,2,4}$ and Jean Paysant ${ }^{3}$
}

\begin{abstract}
Background: Hand rehabilitation needs valid evaluation tools; the 400-point Hand Assessment (HA) is an exhaustive but not standardised tool. The aim of this study was to validate a standardised version of this test.

Methods: A modified version and a standardised prototype was made for this prospective validation study (four centres, three countries). Psychometric properties studied: reliability (intra-rater and inter-rater, standard error of measurement [SEM], minimum detectable change [MDC],internal consistency); content validity, construct validity with Jebsen Taylor hand function test, QuickDASH, MOS-SF 36 and pain; responsiveness, using an anchor-based approach (ROC curve with area under curve, mean response change) with calculation of MCID. For SEM, MDC and responsiveness, QuickDASH was used for comparison.

Results: One hundred and seventy-six patients with hand/wrist injuries were included between May 2013 and February 2015. One hundred and seventy were available for final analysis: 67\% men; mean age $43.4 \pm 13.2$ years; both manual and office workers (46,5\% of each); $37 \%$ had a hand or wrist fracture.

Reliability: ICC intra-rater $=0.967$ [0.938-0.982]; inter-rater $=0.868$ [0.754-0.932]. Distribution-based approach: for 400point HA/QuickDASH: SEM = 3.48/4.52, MDC =9.065/12.53, internal consistency of 400-point HA: Cronbach a $=0.886$. Validity: Content validity was good according to COSMIN guidelines. Construct validity: correlation coefficient: Jebsen-Taylor hand function test $=-0.573$ [ - 0.666-0.464], QuickDASH $=-0.432$ at T0 [ $0.545-0.303],-0.551$ at T3 [-0.648-0.436]; MOS-SF 36 physical component $=0.395$ [0.263-0.513]; no correlation with MOS-SF 36 mental component $=0.142[-0.009+0.286]$ and pain $=-0.166[-0.306+0.018]$.

Responsiveness: Anchor-based approach: AUC $\Delta 400$-point HA $=0.666$ [0.583-0.749], AUC $\Delta$ QuickDASH $=0.556$ [0.466-0.646]. MCID (optimal ROC curve cut-off): 6.07 for 400-point HA, - 2.27 for QuickDASH. MCID with mean response change $+12.034 \pm 9.067$ for 400-point HA and $-8.03 \pm-9.7$ for QuickDASH. The patient's global impression of change was only correlated with the $\Delta 400$-point $H A$.
\end{abstract}

(Continued on next page)

\footnotetext{
* Correspondence: Michel.konzelmann@crr-suva.ch

'Department for Musculoskeletal Rehabilitation, Clinique Romande de Réadaptation suva, avenue du grand champsec, 1950 Sion, Switzerland ${ }^{2}$ Institute for Research in Rehabilitation, Clinique Romande de Réadaptation suva, avenue du grand champsec, 1950 Sion, Switzerland

Full list of author information is available at the end of the article
}

C C The Author(s). 2020 Open Access This article is licensed under a Creative Commons Attribution 4.0 International License, which permits use, sharing, adaptation, distribution and reproduction in any medium or format, as long as you give appropriate credit to the original author(s) and the source, provide a link to the Creative Commons licence, and indicate if changes were made. The images or other third party material in this article are included in the article's Creative Commons licence, unless indicated otherwise in a credit line to the material. If material is not included in the article's Creative Commons licence and your intended use is not permitted by statutory regulation or exceeds the permitted use, you will need to obtain permission directly from the copyright holder. To view a copy of this licence, visit http://creativecommons.org/licenses/by/4.0/. The Creative Commons Public Domain Dedication waiver (http://creativecommons.org/publicdomain/zero/1.0/) applies to the data made available in this article, unless otherwise stated in a credit line to the data. 
(Continued from previous page)

Conclusions: The 400-point HA standardised version has good psychometric properties. For responsiveness, we propose an MCID of at least 12.3/100. However, these results must be confirmed in other populations and pathologies.

Trial registration: This study was retrospectively registered into ISCTRN registry (Number ISRCTN25874481) the 07/ 02/2019.

Keywords: Hand- 400-point hand assessment- functional evaluation-rehabilitation-MCID-MDC-reliability-validity

\section{Background}

Injuries of the hand and wrist are very common and are responsible for significant costs which comprise direct costs (surgery, rehabilitation) and indirect costs (days off work, compensations). For example, in Switzerland (8 million inhabitants) in 2008, 160,115 injuries of the hand/wrist/fingers occurred costing 419 million Swiss francs (estimation in 2012). They represented 24, $4 \%$ of the total injuries in 2008 in Switzerland [1].

Many of these injuries occurred in the active working population and could lead to permanent sequela and disability. In rehabilitation and particularly in vocational rehabilitation, useful evaluation tools are needed with good psychometric properties to establish a treatment plan based on precise goals, to measure improvements in the rehabilitative process and to measure functional capacities at the end of treatment and to prove treatment efficacy among providers [2]. In rehabilitation, the international classification of functioning (ICF) model is used [3] from the World Health Organization (WHO); it is a biopsychosocial view of functioning organized in two parts. The first part (functioning and disability) comprises body functions and structure, activities and participation. The second part (contextual factors) comprises environmental and personal factors.

A good evaluation process needs to cover all of the ICF dimensions [4-9]. For example, pain is a body function and may be evaluated by a visual analogue scale (VAS), as is range of motion, which is evaluated with a clinical exam and a goniometer. "Activities" refers to the execution of a task or "to do something" and participation reflects the implications to the patient in real life (work and leisure activities for example).

To measure activities and participation, different tools are available. First, the patient-reported outcomes (PRO) have been commonly used for at least 20 years; for the upper limb, the gold standard is the Disabilities Arm Shoulder and Hand (DASH) questionnaire [10]. Many other questionnaires are available [11]. In 2017, Kus [9] published a brief ICF core set for hand conditions (ICF Hand A) with different evaluations for screening all the dimensions of the ICF. To assess activities and participation, they proposed using a functional capacity evaluation (FCE) [12], Moberg pick up test [13] and the
DASH. For patients with a recent injury or surgery, administration of a FCE may not be feasible. Moreover, the FCE is time-consuming and not only focused on the upper limbs. Less time-consuming and more specific evaluation tools are needed. Many hand evaluation tests have become available since 1965 [14]. Despite insufficient psychometric properties [15], the most-used test in the literature is the Jebsen-Taylor hand function test (JTHFT) [16]. It is standardised with seven tasks and easy to administer with norms. It is mainly used in neurology and less used for orthopaedic injuries. Two other tests have good psychometric properties: the Test d'Evaluation des Membres supérieurs des Personnes Agées, or upper-extremity performance test for the elderly (TEMPA) [17-19], and the Sequential Occupational Dexterity Assessment (SODA) [20] and its short version [21], but they were developed for the elderly population (TEMPA) or for patients with rheumatoid arthritis (SODA) and are also used in neurology. Schoneveld [6] recommends using the functional dexterity test [22, 23], which is not exhaustive for all hand function.

A more comprehensive hand function test is required. The 400-point Hand Assessment (400-point HA, bilan 400 points in French) test was designed and validated by a French team of occupational therapists and physical medicine and rehabilitation (PMR) physicians in Nancy, France, from 1985 to 1997 [24-27]. It was inspired by different tests published previously $[16,28,29]$. The item choices were made by the team, based on clinical practice, specifically to cover hand function and to target the overall functional deficit. No patients were involved in the choice of items but they tested the different versions. It's a quantitative and evaluative test used in pathologies of the musculoskeletal system in adults. It covers the first part of the ICF well, with 1 item for body structures, 12 items for body functions and 21 items for activities and participation [30]. There are four tests, each based on 100 points: function of the hand (mobility with 12 movements), strength (five dynamometers), handling and displacement of objects (20 objects), and function with both hands (20 tasks of daily living). The final result is the sum of each test divided by 4 and is expressed as a percentage of 100 . The protocol is well described [26]. The test is unidimensional [25, 31]. A principal 
component analysis was made for the injured hand and showed three factors; each factor explained 44, 10 and $4 \%$ of the total variance, respectively [25]. For the authors, three arguments in favour of test unidimensionality were: the first factor being greater than $20 \%$, only 6 of the 67 items having a weak correlation $(<0.30)$ with first factor, and finally the ratio between the first and second factor being 4.4 (it should be $>2$ ). The results were the same for the non-injured hand and for the total analyses. Cronbach's $\alpha$ value at 0.97 testified to good congruence of the items, and the Rasch model was also consistent with the unidimensionality; only one item was rejected by the U test of Molinar [25]. After removal of some redundant items, the final version of the 400-point HA was published in 1996 [26, 27]. The inter-rater and the test retest reliability were studied and found to be good [24, 31, 32], with intraclass correlation coefficients (ICC) of more than 0.95 and 0.82 , respectively.

The 400-point HA is used mainly in France, the French-speaking part of Belgium and Switzerland, Portugal and in some Spanish-speaking countries. The Portuguese translation is available and was done by a Portuguese occupational therapist (OT) who spoke French. The Spanish version was translated and validated in 2014 by a team of OTs in Chile [31]. For this article, we translated the protocol in English.

Because of non-standardised equipment, a complicated strength test, partial data on construct validity and responsiveness, it was decided to build a modified and standardised version of the 400-point HA and validate it.

The aim of this prospective, multicentre study was to modify and simplify the 400-point HA, to build a standardised prototype and to validate its psychometric properties: reliability (intra- and inter-rater reliability, measurement error, MDC and internal consistency), content and construct validity and responsiveness.

\section{Methods}

\section{Construction of the 400-point HA, version 2}

The first stage of the study was to modify the original version of the 400-point HA. This was done by the team of occupational therapists (Mrs. Gable et al.) of the Institut Régional de Réadaptation, Nancy, France. The modifications were based on the opinion of a team experts. The protocol is available as supplementary material in French, Portuguese and English (see Additional files 1, 2 and 3$)$.

In the first part (mobility of the hand), the wrist flexion/extension was added to give an overall view of the entire function of the hand. Fourteen global movements are tested in this first part.

In the second part, assessing strength, three dynamometers were removed for practical reasons; two of them were outdated (the Collins dynamometer and the vigorimeter), and one was homemade. Administration time should be improved by using only two dynamometers, one for grip strength (Jamar ${ }^{\bullet}$ Dynamometer, at the second notch), and one for pinch strength between the thumb and index finger (Jamar ${ }^{\circ}$ pinch-meter dynamometer). Three measures are made (alternately) on both sides and the mean is calculated. For the norms of the two dynamometers, it was decided to use the Swiss norms published in 2010 [33].

For the third part (handling and displacement of objects) objects were standardised for size and weight. Two objects were modified: a cylinder of $7.5 \mathrm{~cm}$ was added and a trunnion removed (expert team opinion). Twenty actions of prehension are tested.

For the fourth part (function with both hands), the material was also standardised and some minor modifications of objects were done: a $20 \mathrm{~mm}$ nut was removed, and the purse and medication tube with crimped cap were updated (expert team opinion). Twenty bimanual actions are available for this test.

\section{Realisation and production of four prototypes of the 400- point HA}

Four standardised prototypes were built with the help of an engineering school in Nancy, France, for some objects (cube, cylinder). The four prototypes were similar and were given to each study centre.

\section{Protocol of the study (see Fig. 1)}

This was a prospective validation multicentre study involving four centres in three countries. Two French centres (Institut régional de médecine physique et de réadaptation, centre Louis Pierquin, Nancy; centre médical Rocheplane, Saint Martin d'Hères); one Portuguese centre (Hospital particular do Algarve Gambelas, Faro) and one Swiss centre (Clinique romande de réadaptation suva, Sion). The inclusion period was expected to run from May 2013 to May 2014 and the results analysis from May 2014 to September 2014. Two hundred and fifty patients were planned to be included: 100 at Nancy and 50 at each of the other centres. For sample size, no clear scientific recommendations are available [34]. A sample size of approximately 100 is considered sufficient for multivariate analysis [34]. Based on other studies of hand test validations $[15,16,18,20,22]$, the number of planned subjects is greater in this study. For the original version of the 400-point HA, Luquet [25] administered the test to 173 patients.

\section{Inclusion criteria}

Patients over 18 years old, with unilateral traumatic hand impairment (fractures, tendon lesions, wounds, peripheral traumatic neuropathy, Complex Regional Pain 


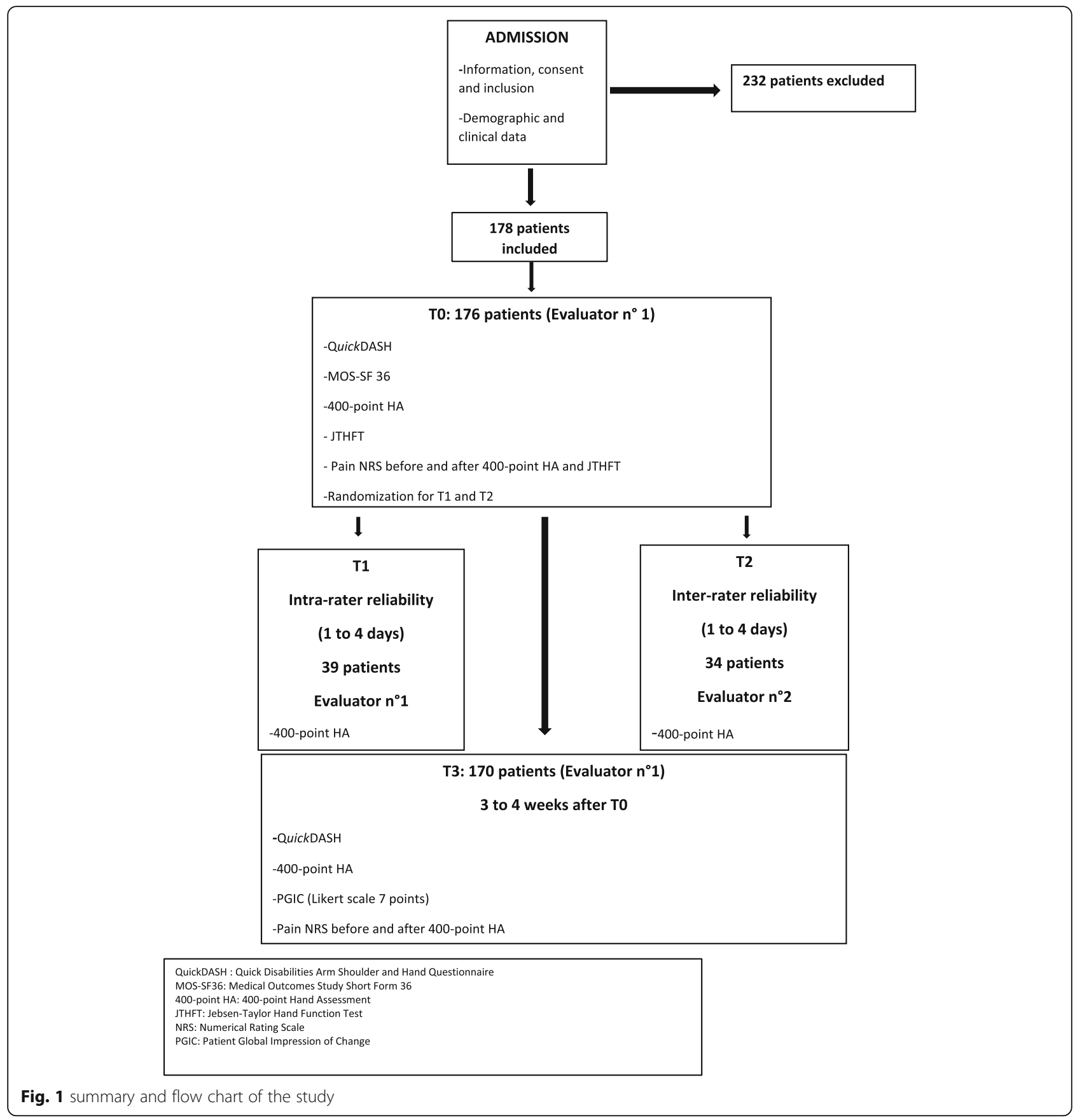

Syndrome [CRPS]) were included, whether hospitalized or not.

Exclusion criteria: Patients under 18 years old, bilateral hand impairment, central neurological impairment, severe psychiatric disorders, patients unable to fulfil the questionnaires or to understand the instructions of the 400-point HA, impossibility to plan the 400-point HA at admission, contraindication for the 400 -point $\mathrm{HA}$ at admission (recent surgery).

Data at admission: Patients were screened for inclusion and exclusion criteria and received an information sheet. Patients who fulfilled the inclusion criteria and were willing to participate signed an informed consent. If the patient met the exclusion criteria or refused to participate, he or she was assigned to the group of excluded patients. The following data were collected: birth date, sex, profession, school education (under nine years or over nine years), pathology and type of lesion, hand dominance, hand side involved, injury date, surgical intervention date, rehabilitation start date, hospitalized or ambulatory treatment, work incapacity, pain numerical rating scale (NRS) maximum and average in the past week. 
The measurement properties of the 400-point HA were planned according to COSMIN guidelines consensus $[35,36]$. Reliability, validity and responsiveness were to be studied.

Reliability reflects "the extent to which scores for patients who have no change are the same for repeated measurement under several conditions". It includes intra-rater and inter-rater reliability, measurement error and internal consistency [36].

\section{Intra- and inter-rater reliability}

Some patients after inclusion and first evaluation (T0) were randomly assessed for intra-rater reliability (T1) or for inter-rater reliability (T2). These patients were not the same. The number of patients for $\mathrm{T} 1$ and $\mathrm{T} 2$ was estimated at 40 patients in each group. Because no international recommendations are available, this number was chosen based on previous studies about the original 400-point HA [31, 32] and on other studies of functional hand tests $[18,20,22]$. The intraclass correlation coefficient (ICC) of the original version of the 400-point HA was good with 20 to 30 patients, so we expected the same result with 40 patients in each group. The 400point HA was administered 1-4 days after T0, by the same evaluator (T1) and by another evaluator (T2). This interval was chosen because no improvement was expected in this short span of time.

For statistical analysis, intra-rater and inter-rater reliability were assessed with the intraclass coefficient correlation (ICC) [37] and the Bland-Altman method [38]. The mean of the two 400-point HA tests (1-4 days) was calculated between $\mathrm{T} 0$ and $\mathrm{T} 1$ for intra-rater reliability, and between $\mathrm{T} 0$ and $\mathrm{T} 2$ for inter-rater reliability. The ICC was considered excellent if $r>0.91$, good if $r$ was between 0.71 and 0.9 , medium between 0.51 and 0.70 , weak between 0.31 and 0.50 and no correlation if $\mathrm{r}<0.30$ [37].

We expected the ICC to be $>0.8$ for each reliability.

\section{Measurement error}

The standard error of measurement (SEM) was measured, which is the variation in scores due to the unreliability of the scale used. A SEM value is based on the standard deviation $(\mathrm{SD})$ of the sample and the reliability of the measurement instrument, expressed as SEM $=\mathrm{SD}$ from the first test $x(\sqrt{ }(1-\mathrm{ICC}))$ [39]. The ICC is the result of the intra-rater reliability of the 400-point HA. Minimum detectable change (MDC) was also calculated. It refers to the least amount of change outside of error that reflects true change by a patient between two time points, rather than a variation in measurement. The formula for MDC is MDC $=1.96 \times$ SEM X $\sqrt{ } 2$. The value 1.96 was chosen to achieve a confidence interval of $95 \%$ [40].

\section{Internal consistency}

Internal consistency refers to "the homogeneity of a measure in terms of how the items of an instrument group together into units" [41]. Internal consistency of the 400-point HA was determined by Cronbach's $\alpha$, which is a general coefficient of homogeneity between items. Values for $\alpha$ can range from zero (no internal consistency) to one (perfect internal consistency). A value above 0.8 is considered acceptable [37]. Cronbach's $\alpha$ was calculated for each test and for the total score of the 400-point HA.

Cronbach's $\alpha$ was hoped to be $>0.8$ for all the tests and for the total 400-point HA.

Validity is "the degree to which a measure instrument measures the construct it purports to measure" [36]. It includes content validity, construct validity and criterion validity.

Content validity is the degree to which the content of an instrument is an adequate reflection of the construct to be measured. Usually it is an expert opinion (the authors) and COSMIN guidelines were used for it [35].

Construct validity is "the degree to which a score of an instrument is consistent with hypotheses based on the assumption that the instrument validly measures the construct to be measured" [36]. It could be studied by testing different hypotheses. For the 400-point HA, which is a hand function assessment, construct validity must be studied with instruments designed for hand function and which comprise activities of daily living in particular.

Two questionnaires were used, the Quick Disabilities Arm Shoulder and Hand questionnaire (QuickDASH) ( [42, 43] and the Medical Outcomes Study-Short Form (MOS-SF) 36 [44-46] in validated French and Portuguese versions; the Jebsen-Taylor hand function test (JTHFT), another hand function test [16]; and the pain numerical rating scale (NRS).

The QuickDASH has been used for many years worldwide, and many translations are available. It is easy to administer and less time-consuming, with only 11 questions, reason why it was chosen. It was administered at $\mathrm{T} 0$ and $\mathrm{T} 3$.

The MOS-SF 36 is a validated questionnaire about quality of life (QOL), with different components. For our study, the physical component (PC) and the mental component $(\mathrm{MC})$ of the questionnaire were used for comparison. The questionnaire was administered at T0.

Because no gold standard test for hand function was available, the JTHFT was chosen as comparator for the 400-point HA. It is standardised, commercialised in French [47] and Portuguese [48] versions and is easy to administer. Each of the seven tests of the JTHFT is timed. The two tests were administered at T0 by very seasoned occupational therapists, six at Nancy, five at 
Saint Martin d'Hères, three at Sion and one at Faro. The protocol at T0 was: NRS from 0 to 10 before administration of the 400-point HA, administration of the 400point $\mathrm{HA}$ in one session (30 to $40 \mathrm{~min}$ ), NRS from 0 to 10 after, rest for $10 \mathrm{~min}$, NRS from 0 to 10 before JTHFT, administration of JTHFT [16] and NRS from 0 to 10 after. For comparison, mean pain was used at T0 after the test.

For statistical analysis, Pearson's correlation coefficients ( $r$ ) was used between the 400-point HA and the QuickDASH, MOS-SF 36 physical component (PC) and mental component (MC) and mean pain NRS at T0. Spearman's correlation coefficients ( $r$ ) were used between the 400-point HA and JTHFT at T0. For statistical analysis, the times of the seven tests were compared to the norms of the JTHFT and summed up for each hand (injured and non-injured) and compared to the score of the 400-point HA. Only the time of the JTHFT and 400-point HA scores for the injured hand was compared.

Correlation was considered excellent if $r>0.91$, good if $\mathrm{r}$ was between 0.71 and 0.9 , medium between 0.51 and 0.70 , weak between 0.31 and 0.50 and no correlation if $\mathrm{r}<0.30$ [37]. Ninety-five percent confidence intervals for the correlation coefficients were calculated by means of Fisher's transformation.

\section{Our hypotheses were}

Expected good correlation between JHFT and 400-point HA

Expected medium correlation between QuickDASH and 400-point HA

Expected weak correlation between MOS-SF 36-PC and 400-point HA

No expected correlation between SF 36 MC and 400point HA

No expected correlation between pain NRS and 400point HA

Criterion validity: In the absence of a reasonable gold standard, COSMIN does not recommend studying this validity [35].

Responsiveness is "the ability of an instrument to detect change over time in the construct to be measured" [36]. No consensus exists for its calculation [49]. The anchor-based approach was chosen which is the method of choice for numerous authors. The anchor used was the level of improvement based on the patients' subjective feelings reported on the Patient Global Impression of Change (PGIC) a Likert scale of seven items [49]. It asks patients how their health status has improved following treatment ( $1=$ worse than ever; $2=$ much worsened; $3=$ slightly worsened, 4 = unchanged, 5 = slightly improved; $6=$ much improved; $7=$ completely improved). The score was treated as a binary outcome (scores of 6 or
7 = "improved" versus scores of 1 through $5=$ "not improved"). Minimal clinically important difference (MCID) of the 400-point HA was estimated using the receiver operating characteristic (ROC) method by comparing patients with and without improvement. The optimal cut-off on the ROC curve was determined by using the optimal Youden's index [50]. The mean change of score according each patient's response level on the PGIC was also calculated. Revicki [51] recommended that the MCID must be based primarily on appropriate patient-based anchors that are correlated at $\geq 0.30$ with the patient-reported outcome. To reinforce the validity of our anchor, the Spearman correlation coefficient was calculated between the PGIC and delta scores. For the ROC curve, an AUC $>0.70$ was expected for the 400point HA [52].

The effect size (ES) and the standardised response mean (SRM) were not calculated because COSMIN guidelines recommended not to use this distributionbased approach for responsiveness [35].

As a comparison, the MCID of the QuickDASH was also estimated. The ICC for the test-retest of the DASH was 0.95 [42] for the French version.

All calculations were performed using the Stata 16.0 statistical package for Windows.

\section{Interventions between TO and T3}

Therapeutic interventions were different in centres and were not standardised. All the patients in all centres were treated by an occupational therapist (OT). Some patients were treated every day (for example, inpatients from Sion, Nancy and Saint-Martin D'Hères centres), some others two or three times a week (outpatients from Faro, Nancy and Saint-Martin d'Hères). Other therapies were used for inpatients: physiotherapy and group therapy (balneotherapy, fitness, and adapted physical activities) centered not only on hand pathologies but on general physical well-being. Pain medications were taken if necessary.

Data summary All the data were summarised in an anonymous booklet with a number for each patient. When the booklet was finished, each centre sent it to the Clinique Romande de Réadaptation suva in Switzerland and the booklet was verified and scanned directly in an anonymous Excel file.

Ethics The current study was approved by the local ethical committee of the four evaluation centres and was conducted in accordance with the Helsinki Declaration. For Clinique Romande de Réadaptation, the Commission Cantonale Valaisanne d'Ethique Médicale ( ${ }^{\circ}$ CCVEM 050/12); for France (two centres) Comité d'Ethique de l'Institut Régional de Médecine Physique et Réadaptation 
and for Portugal Comissao de Etica para a Saude do Hospital Particular de Algarve ( $\left.n^{\circ} 1 / 2013\right)$ gave consent. All patients received an information sheet about the study and signed an informed consent if they were included.

\section{Results}

Descriptive statistics: Patients were included from May 2013 to February 2015; 410 patients were eligible. One hundred and seventy-eight patients were included; 176 were available at T0 and 170 at T3 for the final analysis. Two hundred and thirty-two were excluded for several reasons. The excluded group differed by fewer cases of CRPS and fewer workers than among the included group. The characteristics of the two groups are shown in Table 1. Our patients were mainly men (67\%), middle-aged (median 43 years old), workers (46.5\%) and employees (46.5\%) and in total incapacity to work (82\%). The main lesions were hand or wrist fractures (37\%) and $28 \%$ had an associated CRPS. The characteristics of patients between centres are available in supplementary material (see Additional file 4). The following differences were found: the Faro centre had more women, more employees, fewer cases of CRPS, and more acute patients and 400-point HA results were better at T0 than in other centres. The Saint Martin d'Hères Centre had no CRPS patients. The Sion centre had more workers, a longer interval between injury and T0 and a higher pain level than other centres. The Sion and Nancy centres were relatively similar.

\section{Reliability: results are presented in Fig. 2 and Table 2}

Intra-rater reliability was made for 39 patients between T0 and T1 (mean $=3.23$ days). The 39 patients differed from the others only by their pain at T0 being slightly lower $(p=0.008)$. The ICC was excellent at 0.967 [0.938-0.982] for the total score of the 400-point HA. The ICC for each test of the 400-point HA ranked from 0.974 (test $n^{\circ} 4$ ), 0.972 (test $n^{\circ} 3$ ); 0.810 (test ${ }^{\circ} 2$ ) to 0.752 (test $\left.n^{\circ} 1\right)$. With the Bland-Altman method, the mean difference between tests at $\mathrm{T} 0$ and $\mathrm{T} 1$ was -2.5 , with $95 \%$ upper and lower limits of agreement of -10.33 and 5.32, respectively, including three outliers.

Inter-rater reliability was made for 34 patients between T0 and T2 (mean $=3.35$ days). The 34 patients differed from the others only by maximum and mean pain at T0 which was also slightly lower ( $p=0.088$ and $p=0.0065$ respectively). The ICC was good at 0.868 [0.754-0.932] for the total score of the 400-point HA. The ICC for each test of the 400-point HA ranked from 0.920 (test $n^{\circ} 2$ ), 0.815 (test $n^{\circ} 1$ ) and 0.784 (test $n^{\circ} 3$ ) to 0.700 (test $n^{\circ} 4$ ). With the Bland-Altman method, the mean difference between tests at $\mathrm{T} 0$ and $\mathrm{T} 2$ was -1.8 , with 95\% upper and lower limits of agreement of -18.68 and 15.05 , including two outliers.

\section{Measurement error}

SEM and MDC were calculated for all centres and by each centre. The results of all centres were available for 169 patients. For the 400-point HA SEM, the ICC of 0.967 (intra-rater reliability) was taken. The SEM was at 3.48, the MDC at 9.65. There were some disparities between centres for SEM and MDC. For the QuickDASH SEM, the ICC of 0.95 was taken [42]. The SEM was at 4.52, the MDC at 12.05 .

\section{Internal consistency}

The Cronbach's $\alpha$ for the entire 400-point HA was 0.886 and, for each test, it was 0.842 for test $\mathrm{n}^{\circ} 1,0.543$ for test $\mathrm{n}^{\circ} 2,0.968$ for test $\mathrm{n}^{\circ} 3$ and 0.944 for test $\mathrm{n}^{\circ} 4$.

\section{Validity: results are presented in Table 3 Content validity}

COSMIN recommendations [35] about content validity were applied (Box D of recommendations). The 400point HA items were relevant for the construct to be measured (D1) (global functional evaluation of the hand comprising mobility, strength, different prehension, bi manual activities), study population of patients with orthopaedic lesions of the hand (D2) and evaluative purpose of the test (D3). For the comprehensiveness of the items (D4), we think that all the relevant aspects of the construct were covered by the items and the domains. As is said in the introduction, ICF domains of body functions and structure, activities and participation were covered by the 400-point HA [30]. The conceptual basis of the 400-point HA was to appreciate global hand functional capacity and to target parameters that are responsible of the global deficit. We think that this aim was achieved with this test. For the last requirements (D5), we think that the design and methods of the study have no important flaws. We conclude that the 400-point HA has good content validity.

\section{Construct validity}

At T0, 173 patients were available.

With JTHFT, the correlation with the total 400-point HA score was medium at $-0.573[-0.666-0.464]$. Of the four tests of the 400-point HA, the second test (force) correlated the best with the JTHFT, also with a medium correlation. The three others had a weak correlation.

With QuickDASH, the correlation at T0 was weak at $-0.432[-0.545-0.303]$ and at T3 was medium at $0.551[-0.648-0.436]$.

With the MOS-SF 36 physical component (PC), the correlation was weak at 0.395 [0.263-0.513]. 
Table 1 Characteristics of the two populations

\begin{tabular}{|c|c|c|}
\hline & PATIENTS INCLUDED & PATIENTS EXCLUDED \\
\hline Number of patients (total) & 176 & 235 \\
\hline Nancy & 66 & 88 \\
\hline Sion & 58 & 81 \\
\hline Saint Martin d'Hères & 32 & 49 \\
\hline Faro & 20 & 17 \\
\hline Causes of exclusion & NA & \\
\hline Age $<18$ years & & $6(2.5 \%)$ \\
\hline Bilateral injury & & $39(16.6 \%)$ \\
\hline Central neurological injury & & $4(1.7 \%)$ \\
\hline Psychiatric comorbidity & & $8(3.4 \%)$ \\
\hline Planning impossible & & $49(20.9 \%)$ \\
\hline Fluency in French or Portuguese & & $39(16.6 \%)$ \\
\hline Refusal & & $8(3.4 \%)$ \\
\hline Contra indication to 400 -point $\mathrm{HA}$ & & $82(34.9 \%)$ \\
\hline Men & $118(67.05 \%)$ & $157(66.8 \%)$ \\
\hline Women & $58(32.95 \%)$ & $78(33.2 \%)$ \\
\hline $\begin{array}{l}\text { Age (years) } \\
\text { Median }+- \text { SD }\end{array}$ & $43.4+-13.2$ & $44.3+-15.3$ \\
\hline \multicolumn{3}{|l|}{ School education $(n=176)$} \\
\hline$<9$ years & $50(28.4 \%)$ & \\
\hline$>9$ years & $126(71.6 \%)$ & \\
\hline \multicolumn{3}{|l|}{ Employment status $(n=176)$} \\
\hline Employee & $135(76.7 \%)$ & \\
\hline Unemployment & $25(14.3 \%)$ & \\
\hline Retired & $12(6.8 \%)$ & \\
\hline Student & $2(1.1 \%)$ & \\
\hline Housewife & $2(1.1 \%)$ & \\
\hline \multicolumn{3}{|l|}{ Occupation $(n=172)$} \\
\hline Worker/farm worker & $80(46.5 \%)$ & $80(34 \%)^{a}$ \\
\hline Employee (office) & $80(46.5 \%)$ & $70(29.8 \%)^{a}$ \\
\hline Manager/self employed & $6(3.5 \%)$ & $16(6.8 \%)$ \\
\hline Other & $6(3.5 \%)$ & $58(29.4 \%)^{a}$ \\
\hline \multicolumn{3}{|l|}{ Work capacity $(n=175)$} \\
\hline $0 \%$ & $143(81.7 \%)$ & \\
\hline Partial & $8(4.6 \%)$ & \\
\hline Complete & $24(13.7 \%))$ & \\
\hline \multicolumn{3}{|l|}{ Hand dominance $(n=176)$} \\
\hline Right handed & $162(92 \%)$ & \\
\hline Left handed & $12(6.8 \%)$ & \\
\hline Ambidextrous & $2(1.2 \%)$ & \\
\hline \multicolumn{3}{|l|}{ Hand injured $(n=176)$} \\
\hline Right & $93(52.8 \%)$ & \\
\hline Left & $83(47.2 \%)$ & \\
\hline
\end{tabular}

Diagnostic $(n=176)$ 
Table 1 Characteristics of the two populations (Continued)

\begin{tabular}{|c|c|c|}
\hline & PATIENTS INCLUDED & PATIENTS EXCLUDED \\
\hline Fracture hand/wrist & $65(36.9 \%)$ & $62(26.4 \%)$ \\
\hline Sprain/luxation & $18(10.3 \%)$ & $22(9.4 \%)$ \\
\hline Isolated tendon & $23(13 \%)$ & $25(10.6 \%)$ \\
\hline Complex lesion (> 3 structures) & $26(14.8 \%)$ & $49(20.8 \%)$ \\
\hline Others & $44(25 \%)$ & 77 (32.8\%) \\
\hline \multicolumn{3}{|l|}{ CRPS $(n=176)$} \\
\hline Yes & $50(28.4 \%)$ & $36(15.3 \%)^{\mathrm{a}}$ \\
\hline No & $126(71.6 \%)$ & 199 (84.7\%) \\
\hline $\begin{array}{l}\text { Interval between injury and T0 }(n=163) \\
\text { Days median }+- \text { SD }\end{array}$ & $233+-460.3$ & \\
\hline $\begin{array}{l}\text { Number of surgery }(\mathbf{n}=\mathbf{1 7 6}) \\
\text { median }+-\mathrm{SD}\end{array}$ & $1+-1.22$ & \\
\hline $\begin{array}{l}\text { Interval between last surgery and T0 }(n=143) \\
\text { Days Median }+-S D\end{array}$ & $109+-175.2$ & \\
\hline $\begin{array}{l}\text { Pain at T0 before test }(n=175) \\
\text { median }+-S D\end{array}$ & & NA \\
\hline Average pain & $3+-2.11$ & \\
\hline Maximum pain & $5+-2.76$ & \\
\hline $\begin{array}{l}\text { Pain at T3 before test }(n=170) \\
\text { median }+- \text { SD }\end{array}$ & & NA \\
\hline Average pain & $3+-2.17$ & \\
\hline Maximum pain & $5+-2.85$ & \\
\hline $\begin{array}{l}\text { QuickDASH/100 } \\
\text { median +- SD }\end{array}$ & & NA \\
\hline T0 $(n=176)$ & $52.2+-20.2$ & \\
\hline T3 $(n=170)$ & $40.9+-19.5$ & \\
\hline $\begin{array}{l}\text { 400-point HA (in \%) at T0 }(\mathbf{n}=\mathbf{1 7 6}) \\
\text { median +- SD }\end{array}$ & & NA \\
\hline Total score & $60.3+-19.2$ & \\
\hline Test $n^{\circ} 1$ & $63.8+-16.9$ & \\
\hline Test $n^{\circ} 2$ & $48.8+-25.3$ & \\
\hline Test $n^{\circ} 3$ & $60+-25.5$ & \\
\hline Test $n^{\circ} 4$ & $65+-21.9$ & \\
\hline $\begin{array}{l}\text { 400-point HA (in \%) at T3 }(\mathbf{n}=\mathbf{1 7 0}) \\
\text { median }+- \text { SD } \\
\text { Total score }\end{array}$ & $72.6+-18.7$ & NA \\
\hline Test $n^{\circ} 1$ & $71.9+-16.9$ & \\
\hline Test $n^{\circ} 2$ & $57.1+-24.9$ & \\
\hline Test $n^{\circ} 3$ & $80.8+-22.9$ & \\
\hline Test $n^{\circ} 4$ & $83.3+-21.05$ & \\
\hline
\end{tabular}

SD standard deviation, CRPS complex regional pain syndrome, QuickDASH Quick Disabilities Arm Shoulder and Hand, 400-point HA 400-point Hand Assessment, NA not applicable

a Statistically significant difference

With the MOS-SF 36 mental component (MC), the correlation was at $0.142 \quad[-0.009+0.286] \quad$ (no correlation).

With mean pain, the correlation was at $-0.166[-$ 0.306-0.018] (no correlation).

\section{Responsiveness: results are presented in Table 4 and}

Fig. 3

Anchor-based approach

Patients were categorised in two groups, according to their answers to the anchor question: 103 patients 


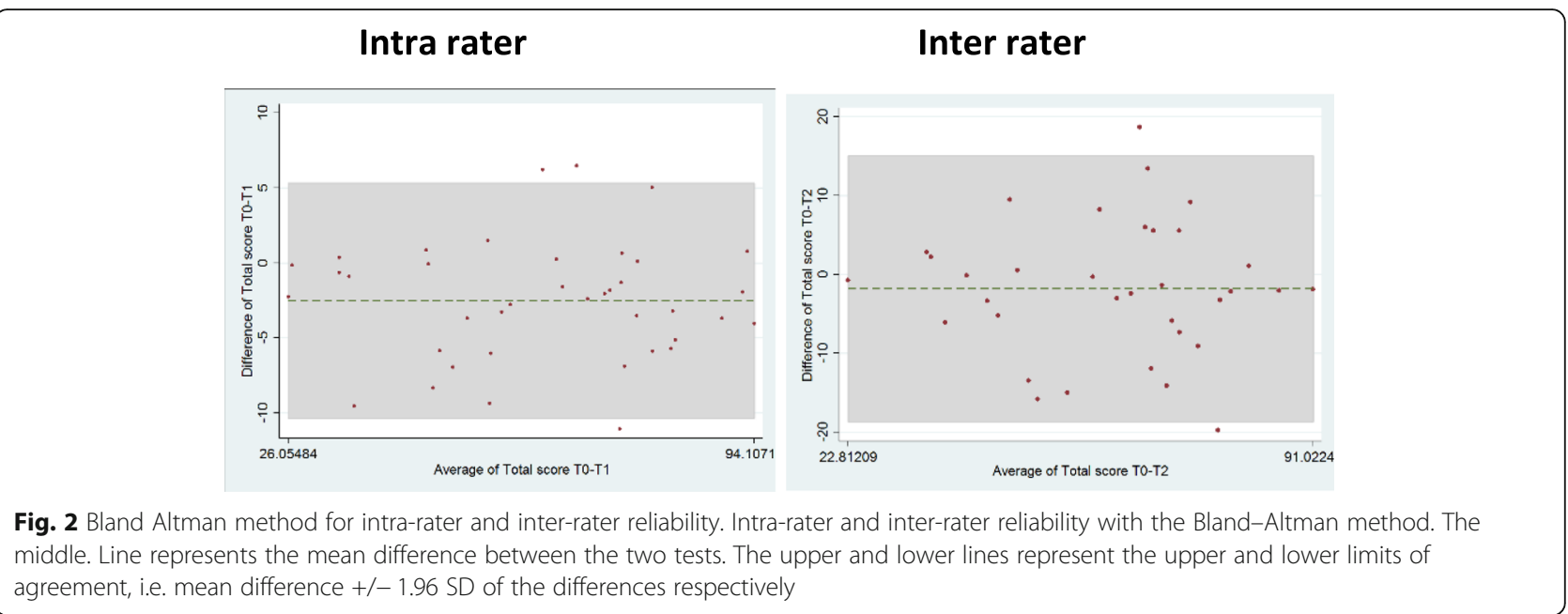

(60.6\%) reported an improvement (i.e., much improved or completely improved, according to the PGIC), and 67 patients (39.4\%) reported no improvement.

ROC curves for the progression of the scores $(\Delta-400$-point HA T0-T3 and $\Delta$-QuickDASH T0-T3) showed an AUC of $0.666[0.583-0.749]$ for the $\Delta-400$-point $\mathrm{HA}$ and 0.556 [0.466-0.646] for the $\Delta$-QuickDASH (see Figure n³).

The MCID was estimated by the optimal ROC cut-off value at 6.07 for the 400 -point HA (sensitivity: $72.82 \%$ and specificity 53.03\%) and -2.27 for the QuickDASH (sensitivity: $91.26 \%$ and specificity $16.42 \%$ ).

In the improved group, the $\Delta-400$-point $\mathrm{HA}$ and $\Delta$ QuickDASH were $+12.34 \pm 9.67$ and $-8.3 \pm 9.7$, respectively. In the unimproved group, the $\Delta-400$-point $\mathrm{HA}$ and $\Delta$-QuickDASH were $5.63 \pm 9.26$ and $-3.2 \pm 9.8$, respectively. The PGIC was correlated with the $\Delta-400$-point HA (Spearman rho $=0.310$ ) and not correlated with the $\Delta$-QuickDASH (Spearman rho $=0.110)$.

\section{Discussion}

The purpose of this prospective study was to validate a standardised and modified version of the 400-point HA. This test has good psychometrics properties, similar to the original 400 -point HA. Our hypothesis for both intra-rater and inter-rater reliabilities and internal consistency were confirmed. The content validity was good and the 400-point HA covers well the most important hand functions (mobility, strength, prehension and object displacement and bi manual activities) and the first part of ICF (body functions and structures, activities and participation) which are very important in rehabilitation. For construct validity, four hypotheses from the five suggested were strictly fulfilled, while the last one was partly verified. Lastly we were able to propose an MCID which is a very important element for daily practice. Of the ten hypotheses put forward, eight were fulfilled (80\%). Due to the large number of hypotheses that were tested, we cannot exclude that some observed associations were actually type 1 errors. Nevertheless, given the large observed values and the reasonable width of confidence intervals, we are confident about the validity of our results.

In our opinion, this modified version of the 400-point $\mathrm{HA}$ is more comprehensive than other existing instruments $[13,14,16,17,20,22,28,29,53]$ for hand function evaluation.

We resume in Table 5, according to Rudman [41] the properties of the 400-point HA.

The discussion for each psychometric properties is developed here below.

\section{Reliability}

Intra-rater reliability was excellent. Only test 1 (mobility) had an ICC <0.8. Gable [32], with 30 patients, also

Table 2 Reliability of 400-point HA (all centers)

\begin{tabular}{|c|c|c|c|c|c|c|c|}
\hline & $\begin{array}{l}\text { INTRA RATER } \\
(\boldsymbol{n}=39)\end{array}$ & $\begin{array}{l}\text { CONFIDENCE INTERVAL } \\
95 \%\end{array}$ & $\begin{array}{l}\text { INTER RATER } \\
(\boldsymbol{n}=34)\end{array}$ & $\begin{array}{l}\text { CONFIDENCE INTERVAL } \\
95 \%\end{array}$ & INTERNAL CONSISTENCY & SEM & MDC \\
\hline TOTAL SCORE 400-point HA & 0.967 & [0.938-0.982] & 0.868 & {$[0.754-0.932]$} & 0.886 & 3.48 & 9.65 \\
\hline TEST $n^{\circ} 1$ & 0.752 & {$[0.577-0.861]$} & 0.815 & [0.663-0.803] & 0.842 & NA & NA \\
\hline TEST $n^{\circ} 2$ & 0.810 & {$[0.668-0.895]$} & 0.920 & {$[0.847-0.959]$} & 0.543 & NA & NA \\
\hline TEST $n^{\circ} 3$ & 0.945 & {$[0.899-0.971]$} & 0.784 & [0.613-0.885] & 0.968 & NA & NA \\
\hline TEST $n^{\circ} 4$ & 0.974 & {$[0.952-0.986]$} & 0.700 & {$[0.481-0.837]$} & 0.944 & NA & NA \\
\hline
\end{tabular}


Table 3 Construct validity of 400-point HA

\begin{tabular}{|c|c|c|c|}
\hline Variable & Correlation & Confidence interval 95\% & $\boldsymbol{P}$ value \\
\hline Total score of 400 -point HA vs total score JHFT at T0 & -0.573 & {$[-0.666-0.464]$} & $<0.001$ \\
\hline Score test $n^{\circ} 1$ of 400 -point HA vs total score JHFT & -0.441 & {$[-0.554-0.312]$} & $<0.001$ \\
\hline Score test $n^{\circ} 2$ of 400 -point HA vs total score JHFT & -0.560 & {$[-0.655-0.448]$} & $<0.001$ \\
\hline Score test $n^{\circ} 3$ of 400 -point HA vs total score JHFT & -0.481 & {$[-0.588-0.358]$} & $<0.001$ \\
\hline Score test $n^{\circ} 4$ of 400 -point HA vs total score JHFT & -0.445 & {$[-0.557-0.317]$} & $<0.001$ \\
\hline Total score 400 -point HA vs total score QuickDASH & $\begin{array}{l}\text { At T0-0.432 } \\
\text { At T3-0.551 }\end{array}$ & $\begin{array}{l}{[-0.545-0.303]} \\
{[-0.648-0.436]}\end{array}$ & $\begin{array}{l}<0.001 \\
<0.001\end{array}$ \\
\hline Total score 400 -point HA vs MOS-SF 36 PC at T0 & 0.395 & {$[0.263-0.513]$} & $<0.001$ \\
\hline Total score 400 -point HA vs MOS-SF36 MC at T0 & 0.142 & {$[-0.009+0.286]$} & 0.024 \\
\hline Total score 400 -point HA vs mean pain at TO & -0.166 & {$[-0.306-0.018]$} & 0.028 \\
\hline
\end{tabular}

Correlations: Spearman was use for correlations between 400-point HA and JHFT, 400-point HA and mean pain (distribution not normal). Pearson was used for 400-point HA and QuickDASH and MOS-SF 36 MCS/PCS

JHFT = Jebsen Hand Function Test

400-point $\mathrm{HA}=400$-point Hand Assessment

QuickDASH = Quick Disabilities Arm Shoulder Hand

MOS-SF36MCS/PCS = Medical Outcomes Study Short Form 36 Mental Component Score/Physical Component Score

found an ICC $>0.90$ for the total score of the original 400-point HA carried out twice the same day by three evaluators. Naranjo-Olguin for the Spanish version of the 400-point HA [31] found an intra-rater correlation of 0.99. In our study, the two evaluations were performed on average within a time window of 3 days. We chose this interval because no improvement was expected within this short period. Nevertheless, according to the Bland-Altman method, the results at $\mathrm{T} 1$ were a little bit different than at T0, with a deviation from zero. However this difference was negligible $(-2,5$ points) and smaller than the SEM and MDC. A learning bias from the patients' side between T0 and T1 may explain this difference within a short period of 3 days.

Inter-rater reliability was good but less than previous findings of the original version. Gable [24], with 85 patients, found an ICC ranging from 0.90 to 0.97 , depending on the specialization of the different evaluators (physiotherapists, occupational therapists). In a second study [32], the same group found an ICC superior to 0.95. Naranjo-Olguin in the Spanish version of the 400point HA [31] found an inter-rater correlation of 0.98 . Generally, test $\mathrm{n}^{\circ} 4$ is the most difficult to quote because of the complexity of the activities which requires both hands. Good experience of the evaluators is necessary for the judgement [32]. In our study, 16 couples of very seasoned occupational therapists (OT) were involved in the inter-rater reliability. This could explain a lesser ICC in our study compared to the previous studies which involved a maximum of 2 or 3 different evaluators.

\section{Measurement error}

According to Beaton and Schmitt [54, 55], we used the method with the SEM to calculate the MDC. These authors stipulated that a higher MDC than SEM is expected. Both SEM and MDC depend on intra-rater reliability. The higher the intra-rater ICC is, the lower the SEM and MDC will be. In our study, the intra-rater ICC is very high with a value of 0.967 . The MDC of the 400-point HA was 2.7 fold higher than the SEM. As a comparison in the validation of the original version of the 400-point HA, in 56 patients we found an SEM of 4.29 and an MDC at 11.9, which are very similar to the current values (unpublished personal data).

For the QuickDASH, as expected, the MDC was also higher than the SEM. Our results are in accordance with previous studies with various pathologies of the upper

Table 4 Responsiveness of 400-point HA and QuickDASH

\begin{tabular}{lll}
\hline & $\begin{array}{l}400-\text { point HA } \\
\text { total score }\end{array}$ & QuickDASH \\
\hline AUC $_{\text {roc }}$ & 0.666 & 0.556 \\
[Confidence interval of $\mathbf{9 5 \%}]$ & {$[0.583-0.749]$} & {$[0.466-0.646]$} \\
MCID ROC curve cut off & 6.07 & -2.27 \\
MCID mean response change in improved group & $12.3+-9.67$ & (Sensitivity: $91.26 \%$ and specificity $16.42 \%)$ \\
\hline
\end{tabular}

AUC Area Under Curve

ROC Receiver Operating Characteristic

MCID Minimum Clinically Important Difference 

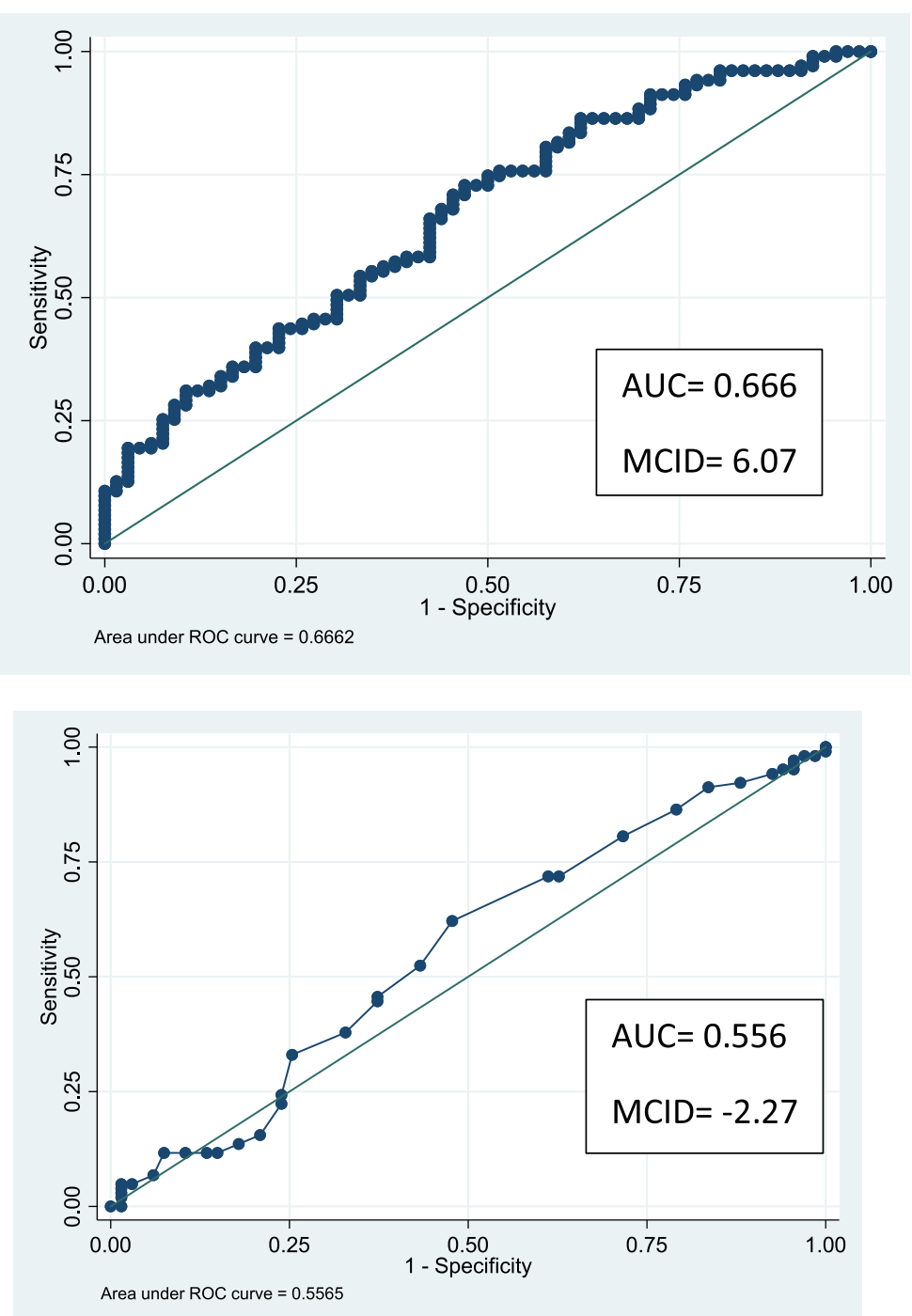

Fig. 3 MCID Anchor based approach with ROC curve and AUC of 400-point HA and QuickDASH. ROC curve of 400-point HA. ROC curve of QuickDASH. ROC: Receiver Operating Characteristic. AUC: Area Under Curve

limb [56, 57], but less than the MDC found in the Smith-Forbes study [58] only for hand pathologies.

\section{Internal consistency}

Cronbach's $\alpha$ of the 400-point HA was lower than the ones from the original French(0.97) [25] and the Spanish versions (0.98) [31]. This could be explained by a low Cronbach's $\alpha$ observed for the second test (strength). In the original version, the strength was measured using five different dynamometers which evaluated quite similar grip capacities. Thus the result was more homogeneous. In the present version, only two dynamometers were used, and they measured two different types of grips (entire hand and thumb-index pinch). From a statistical point of view, the assessment with more items is likely to demonstrate higher internal consistency.

A Cronbach's $\alpha$ of more than 0.80 is considered as good [37], and a value of 0.88 seems sufficient to avoid redundancy of the items. We are very confident of our results because the original 400-point HA was well developed and validated in 1996 and its unidimensionality was confirmed [25].

\section{Validity}

\section{Content validity}

For content validity, all the COSMIN guidelines (Box D) [35] were fulfilled. The 400-point HA is a descriptive and evaluative instrument that covers the hand function well, except for sensitivity. The ICF items not covered by 
Table 5 summarize of the evaluation framework of 400 point HA according to Rudman and Hannah [41]

\begin{tabular}{ll}
\hline CATEGORIES & 400 point HA \\
\hline $\begin{array}{l}\text { Category } 1 \text { : Clinical utility } \\
\text { A) Clinical applicability }\end{array}$ & \\
a. Type of results & Quantitative and qualitative \\
b. Type of tasks & Covers CIF (21 items for activities and participation) \\
$\begin{array}{l}\text { i. Representative of ADL } \\
\text { ii. Unilateral }\end{array}$ & Yes \\
iii. bilateral & Yes \\
c. Administration method & Observation \\
d. Interpretation of results & Comprehensive: four sub-tests (mobility, strength, prehension and displacement of objects, bi manual function), \\
B) Specificity & quality of tasks, percentage of each sub-tests compare to the non-injured hand. \\
C) Availability & Orthopaedics hand injury or pathology, adults \\
a. Prefabricated & \\
b. Public domain & Yes \\
c. Language & Fes \\
d. Cost & In progress
\end{tabular}

\section{D) Time demands}

a. Administration/scoring/ interpretation

30 to 45 minutes

b. Training for evaluator Yes, important at the beginning

\section{E) Acceptability to patients}

a. Purpose understand by Yes patients
b. Appropriate for adults
c. Language
d. Cultural applicability

Yes

French/Portuguese/Spanish/English

Yes used in French and Spanish speaking countries, in Portugal for more than 10 years

\section{Category 2 : Standardization}

A) Instructions
a. Administration
b. Scoring
c. Interpretation

B) Equipment prefabricated
Yes very precise manual

Yes very precise manual

No, the comparison is with the non-injured hand which is considered the normal hand

Yes

Category 3 ; Purpose
A) Descriptive
Yes (comparison with the normal hand)
B) Evaluative
Yes (can be done at the beginning and at the end of the therapy)
C) Predictive
No

\section{Category 4 : Psychometric properties}

A) Items construction

B) Reliability
a. Inter-rater
b. Intra-rater
c. Test-retest
d. Internal consistency

Broad range of items, evaluative and descriptive, items selection by Rasch analysis and principal component analysis
ICC at 0.868
ICC at 0.96
No for this version
Good Cronbach a at 0.886 
Table 5 summarize of the evaluation framework of 400 point HA according to Rudman and Hannah [41] (Continued)

\begin{tabular}{|c|c|}
\hline CATEGORIES & 400 point HA \\
\hline \multicolumn{2}{|l|}{ C) Validity } \\
\hline a. Content & Yes COSMIN recommendations fulfilled \\
\hline b. Construct & $\begin{array}{l}\text { Medium correlation with JHFT (-0.573), weak to medium correlation with QuickDASH }(-0.432 \text { to }-0.559) \text {, weak } \\
\text { correlation with MOS-SF } 36 \text { PC (0.395), no correlation with MOS-SF36 MC and mean pain }(0.142 \text { and }-0.166 \\
\text { respectively) }\end{array}$ \\
\hline c. Criterion & Not applicable no gold standard \\
\hline D) Responsiveness & MCID of 12 points proposed in our study population, not study in other populations \\
\hline \multicolumn{2}{|l|}{ E) Norms } \\
\hline a. Availability & $\begin{array}{l}\text { Yes for the second sub-test (strenght) otherwise the normality is the non-injured hand which is } 100 \% \text { by } \\
\text { definition. If the two hands are injured two } 400 \text { point HA should be done }\end{array}$ \\
\hline b. Quality & Swiss strength norms for adults \\
\hline $\begin{array}{l}\text { Category } 5: \text { patient's } \\
\text { perspective }\end{array}$ & $\begin{array}{l}\text { Not addressed in the development } \\
\text { Can be evaluated with other instruments like questionnaires }\end{array}$ \\
\hline
\end{tabular}

the 400-point HA may be evaluated by a specific test (for example sensitivity) or by questionnaires: QuickDASH or Brief Pain Inventory, for instance.

\section{Construct validity}

For the construct validity we explored the correlation between the 400-point HA and the JTHFT. In this study, the correlation can be considered as medium, which was not as good as expected. This result could be explained by the fact that the 400-point HA explores the entire hand function, whereas the JTHFT mainly assess unilateral tasks involving direct manipulation and use of tools with seven tests. Surprisingly, the third and fourth part of the 400point HA, which are close to the JTHFT, showed a weak correlation. A possible explanation for this, could be the difference in the number of tests in the third and fourth parts of the 400-point HA (40 vs. 7), their complexity and a difference of quotation between the two tests. The JTHFT is only based on time of completion with the dominant and non-dominant hands, whereas the 400-point HA is based on the quality of prehension and displacement of objects by the injured hand and movement quality for the third and fourth tests respectively (the uninjured hand is the comparator.) Time is only informative; it is not used for the calculation of the final score. Our study is the first to make a comparison between the 400-point HA and the JHFT. In the field of locomotor apparatus, no other studies are available for comparison.

The correlation with the QuickDASH was weak at T0 but medium at T3. Similarly, correlation with the MOSSF 36 PC at T0 was weak. These results were expected and in accordance with our hypotheses because the QuickDASH and MOS-SF36 are self-assessment outcomes (subjective) and the 400-point HA is observational and tends therefore to be more objective. As expected, the correlation was slightly better with the specific questionnaire for the upper limb (Quick DASH) than with the MOS-SF36 PC, which is a more generic tool. These results are in accordance with a previous study from our group. In 2011 [59], we found a weak correlation (-0.388) between the 400-point HA and the Patient Rated Wrist Evaluation (PRWE) questionnaire in 30 patients with various wrist disorders; we also found a weak correlation $(-0.491)$ between the original version of 400-point HA and the DASH questionnaire in 56 patients with various hand pathologies (unpublished personal data).

As expected, there was no correlation of the 400-point $\mathrm{HA}$ with the MOS-SF $36 \mathrm{MC}$ and pain, as these are not evaluated by the 400-point HA.

Construct validity is an evolving property which improves along the way with the successive studies [37]. Therefore the validation process never really ends.

\section{Responsiveness}

The 400-point HA has an acceptable responsiveness and we are able to propose an MCID.

First using the ROC curve: for the 400-point HA, the AUC was acceptable, but for the QuickDASH, it was poor. Terwee [52] considered an AUC of at least 0.70 to be adequate. Despite that limit, we calculated an MCID with the optimal cut-off of the ROC curve of 6.07 for the 400-point HA and - 2.27 for the QuickDASH.

Some disparities of AUC between centres were observed in our study and may explain these disappointing results. Three centres had an AUC of 0.76, 0.69 and 0.66, respectively, whereas one centre had a discordant result of 0.27 .Fortunately, this centre only included 18 patients at T3. Combined the AUC of the other centres was 0.70. These three centres (Sion, Saint-Martin d'Hères, Nancy) had the same typology of patients (mainly men, aged between 40 and 50 years, manual workers, injuries), with an interval between injury and study of 1 year or more. The fourth centre (Faro) included more women, who were older, with a 
shorter evolution of 4 months and with more benign pathologies (see additional file 4). We verified all the data from this centre. A plausible explanation for this quite low result may come from the fact that from the 18 patients evaluated at T0 and T3, 13 rated 6 or 7 in the PGIC, and five rated 5. This make it difficult to discriminate improved from unimproved patients in this centre.

As commonly observed in multi centre studies, there were a few differences among the four centres that may explain, in part, these discrepancies. One centre was only ambulatory (Faro). Two centres (Nancy and SaintMartin d'Hères) combined hospitalised and ambulatory patients; management of the patients started just after the injury, and whether hospitalised or not, management continued in an ambulatory setting for a long period of time. The last clinic (Sion) was a tertiary centre, with patients hospitalised for 4 to 5 weeks. Another problem that was identified in our study comes from the interpretation of the PGIC. Indeed, the patients were asked to answer about how their health status had improved following treatment. We realized that the question was not precise enough. Patients followed for a long time by the same centre (all except Sion) referred to their situation immediately after the injury and at T3. They did not compare their situations between T0 and T3, which was the initial goal of the study. This probably led to underestimation of the MCID (around one point in these centres) and thus a lower AUC. An example to illustrate our interpretation, a few patients could have answered "much improved" to the PGIC at T3 (in comparison to their status immediately after the injury), but only displayed a minor improvement in the 400point HA between T0 and T3. In contrast, in Sion for instance, most of the patients entered months after the injury and their answers to the PGIC were related specifically to the current hospitalization. The AUC was better (0.765), and the MCID was 10.4.

There was a consistency between the four centres for the QuickDASH, for not being able to discriminate between the improved and unimproved patients. The MCID was very far from the MDC. Perhaps the interval between T0 and T3 was not long enough to reveal a change. Kennedy [60], in a review about the QuickDASH, reported that responsiveness was poor to fair in eight over the nine studies selected, and that most of the studies did not report correlations between changed scores or the calculation of the AUC.

Second with the mean change score: This approach compares patients who improved (PGIC $=6$ or 7 ) to the others who did not improve (PGIC from 1 to 5 ). This method is recommended in association with the ROC curve for the determination of the MCID [39, 61]. With this method, the results were more consistent for the 400-point HA (MCID >MDC) in correlation with PGIC.
For most authors $[39,49,61,62]$, the combination of the two methods (ROC curve and mean change score) is the gold standard for MCID determination. With this method, several values of the MCID are calculated. There is no clear consensus about the ratio between MCID and MDC. A few authors recommend that the MCID must be greater than the MDC [54, 61], whereas others suggest the exact opposite $[48,54]$. Factors influencing the MCID are well known: the population studied (age, disease group and severity, treatment), the choice of PGIC, the choice of anchor, the base-values and the direction of change [39, 49]. For our population, the 400-point HA MCID was lower than the MDC when assessed with the ROC curve and superior to the MDC, with mean change score. We thought that for clinical use, values under the MDC should be rejected, so we suggested to keep a value of MCID greater than 12.3 for the 400-point $\mathrm{HA}$ in our population. That proposition was reinforced by the calculation of the correlation between the PGIC and the delta 400-point HA as recommended in the literature [51].

These results must be confirmed in other studies and other pathologies.

\section{Our study has a few limitations}

First, our population was mainly composed of middleaged men and manual workers with injuries. We cannot generalize our results to other populations. Second, the standardised equipment needed was not yet easily available, but it will be commercialised soon. Third, as there is a consensus towards the development of less- time consuming tools, our test could appear relatively long to administer (30 to $40 \mathrm{~min}$ ). In our opinion this should not be a barrier as the 400-point HA covers four tests in one. The 400-point HA needs a long learning curve, but its advantage is that you can focus the treatment on the target deficit and do the test again (or a part of it) after treatment. Finally, the 400-point HA was developed for an inpatient population and seems to be more used in hospitals than in the private practice of occupational therapists. From our experience, we are convinced that once mastered this tool could also be easily used in private practice.

\section{Conclusions}

The standardised version of the 400-point HA has good psychometric properties for our population of injured workers.

The reliability (intra and inter-rater reliability, measurement error, MDC and internal consistency) is the same as the original version.

For validity, content validity is good and construct validity is acceptable, with four to five hypotheses fulfilled completely and one hypothesis partially fulfilled. It has 
to be improved in other studies with other PROs or hand evaluation tests.

For responsiveness, the 400-point HA is sensitive to change, and we propose a minimum MCID of 12.3 points (anchor-based approach) for clinical practice. However, the MCID must be studied in other pathologies, particularly in non-injury lesions (tendinitis, carpal tunnel, repetitive injuries, osteoarthritis, and so on) and in other populations which are not represented in our study.

For interpretation of the results, for daily practice, we recommend to look at the total 400-point HA score which is interesting for evaluation of the overall hand function. Then, with the values of the sub-scores, the clinician can focus the therapy upon one or more deficit (mobility, strength, dexterity or bi manual activities).

\section{Supplementary information}

Supplementary information accompanies this paper at https://doi.org/10. 1186/s12891-020-03303-4.

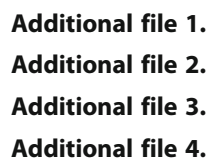

\section{Abbreviations}

ICF: International Classification of Functioning; WHO: World Health Organization; PROs: Patients Reported Outcomes; DASH: Disabilities Arm Shoulder and Hand; QuickDASH: Quick Disabilities Arm Shoulder and Hand; FCE: Functional Capacity Evaluation; JTHFT: Jebsen-Taylor Hand Function Test; TEMPA: Test d'Evaluation des Membres supérieurs des Personnes Agées: SODA: Sequential Occupational Dexterity Assessment; 400-point HA: 400point Hand Assessment; ICC: Intraclass Correlation Coefficient; MOS-SF 36: Medical Outcome Study-Short Form 36; MOS-SF 36 PC: Medical Outcomes Study-Short Form 36 Physical Component; MOS-SF 36 MC: Medical Outcomes Study- Short Form 36 Mental Component; NRS: Numerical Rating Scale; PGIC: Patient Global Impression of Change; MCID: Minimal Clinically Important Difference; ROC: Receiver Operating Characteristic; AUC: Area Under Curve; SEM: Standard Error of Measurement; SD: Standard Deviation; MDC: Minimum Detectable Change; ES: Effect Size; SRM: Standardized Response Mean; CCVEM: Commission Cantonale Valaisanne d'Ethique Médicale; CRPS: Complex Regional Pain Syndrome; PRWE: Patient Rated Wrist Evaluation; MHQ: Michigan Hand Questionnaire; ADL: Activities of Daily Living; FDT: Functional Dexterity Test;

OT: occupational therapist

\section{Acknowledgments}

We thank the occupational therapists who participated at the genesis of the study, included the patients and administered questionnaires, the 400-point HA and the JTHFT. For Saint-Martin d'Hères: Mélanie Chenau, Brigitte Cheviet, Arnaud Gloanec, Pascale Lacombe, Elisabeth Pigeon; for Nancy: Sophie Angel, Emilie Coutant Mulot, Marie-Pierre Gauzelin, Isabelle Gusse, Claire Monereau, Florence Moureau; for Faro: Rosario Simoes; for Sion: Virginie Antonin, Christophe Pelfini, Odile Tomson. Thanks to Dr. Joao Paulo Sousa in Faro, hand surgeon, and the patients. Thanks to Viviane Dufour, research secretary, who scanned the whole booklet of results for the data bank. We thank Chantal Plomb-Holmes MD, for revising English in the manuscript. Procurement information for 400 point HA should be obtained from melanie.chenau@rocheplane.org

\section{Authors' contributions}

MK, CB, FL, CG and JP conceived and designed the work. MK and CB acquired and controlled the data bank. CB conducted the statistical analysis. MK and CB interpreted the data. MK and CB drafted the article. CG, FL and JP reviewed the article critically, and all authors read and approved the final version to be published.

\section{Funding}

There was no external funding or sponsoring in this study.

\section{Availability of data and materials}

The data sets used and/or analysed during the current study are available from the corresponding author upon reasonable request.

\section{Ethics approval and consent to participate}

The current study was approved by the local ethical committees of the fourevaluation centres. For Clinique romande de réadaptation, the "Commission Cantonale Valaisanne d'Ethique Médicale" (n CCVEM 050/12), for France (two centres) and the "Comité d'Ethique de l'Institut Régional de Médecine Physique et Réadaptation"; for Portugal, "Comissao de Etica para a Saude do Hospital Particular de Algarve" ( $n^{\circ} 1 / 2013$ ) gave consent. All patients received an information sheet about the study and signed an informed consent if they were included.

\section{Consent for publication}

The individuals featured in the protocol (supplementary material pages 6 and 22) consented to participate to the study. The consent form did not include the consent to publish, so the two photos were cropped to maintain anonymity. All the other photos were taken by occupational therapists and concerned the hands of occupational therapists.

\section{Competing interests}

The authors declare that they have no competing interests.

\section{Author details}

${ }^{1}$ Department for Musculoskeletal Rehabilitation, Clinique Romande de Réadaptation suva, avenue du grand champsec, 1950 Sion, Switzerland. ${ }^{2}$ Institute for Research in Rehabilitation, Clinique Romande de Réadaptation suva, avenue du grand champsec, 1950 Sion, Switzerland. ${ }^{3}$ Regional institute of physical medecine and rehabilitation, 75 boulevard Lobeau, CS 34209, 54042 Nancycedex, France. ${ }^{4}$ Department of Physical Medicine and Rehabilitation,Orthopaedic Hospital, Lausanne University Hospital, Avenue Pierre Decker, 1011 Lausanne, Switzerland.

Received: 14 February 2019 Accepted: 21 April 2020

Published online: 20 May 2020

\section{References}

1. Ruf O: Suva Abteilung Versicherungstechnik (2014) Unfallstatistik UVG 20082012. Suva, Luzern.

2. Stiens SA, Haselkorn JK, Peters DJ, Goldstein B. Rehabilitation intervention for patients with upper extremity dysfunction: challenges of outcome evaluation. Am J Ind Med. 1996;29(6):590-601.

3. Organization WH: International Classification of Functioning, Disability and Health: ICF: World Health Organization; 2001.

4. Cieza A, Geyh S, Chatterji S, Kostanjsek N, Ustun B, Stucki G. ICF linking rules: an update based on lessons learned. J Rehabil Med. 2005;37(4):212-8.

5. Xiong T, Hartley S. Challenges in linking health-status outcome measures and clinical assessment tools to the ICF. Adv Physiotherapy. 2008;10(3):152-6.

6. Schoneveld K, Wittink H, Takken T. Clinimetric evaluation of measurement tools used in hand therapy to assess activity and participation. J Hand Ther. 2009;22(3):221-36.

7. Dubert T. Outcome measurements in hand and upper limb surgery. Chir Main. 2014;33(4):235-46.

8. Van De Ven-Stevens LA, Graff MJ, Selles RW, Schreuders TA, Van Der Linde $\mathrm{H}$, Spauwen PH, Geurts AC. Instruments for assessment of impairments and activity limitations in patients with hand conditions: A European Delphi study. J Rehabil Med. 2015;47(10):948-56.

9. Kus S, Dereskewitz C, Coenen M, Rauch A, Rudolf KD. Consortium Lighthouse Project H: International Classification of Functioning, Disability 
and Health: development of an assessment set to evaluate functioning based on the Brief ICF Core Set for Hand Conditions - ICF HandA. J Hand Surg Eur Vol. 2017:42(7):731-41.

10. Hudak PL, Amadio PC, Bombardier C. Development of an upper extremity outcome measure: the DASH (disabilities of the arm, shoulder and hand) [corrected]. The Upper Extremity Collaborative Group (UECG). Am J Ind Med. 1996:29(6):602-8

11. Badalamente M, Coffelt L, Elfar J, Gaston G, Hammert W, Huang J, Lattanza L, Macdermid J, Merrell G, Netscher D. Measurement scales in clinical research of the upper extremity, part 2: outcome measures in studies of the hand/wrist and shoulder/elbow. J Hand Surg. 2013;38(2):407-12.

12. Isernhagen SJ. Functional capacity evaluation: Rationale, procedure, utility of the kinesiophysical approach. J Occup Rehabil. 1992;2(3):157-68.

13. $\mathrm{Ng} \mathrm{CL}, \mathrm{Ho} D D$, Chow S. The Moberg pickup test: results of testing with a standard protocol. J Hand Ther. 1999;12(4):309-12.

14. Carroll D. A quantitative test of upper extremity function. J Chronic Dis. 1965;18(5):479-91.

15. Sears ED, Chung KC. Validity and responsiveness of the Jebsen-Taylor Hand Function Test. J Hand Surg Am. 2010;35(1):30-7.

16. Jebsen RH, Taylor N, Trieschmann RB, Trotter MJ, Howard LA. An objective and standardized test of hand function. Arch Phys Med Rehabil. 1969;50(6): 311-9.

17. Desrosiers J, Hébert R, Bravo G, Dutil É. Upper extremity performance test for the elderly (TEMPA): normative data and correlates with sensorimotor parameters. Arch Phys Med Rehabil. 1995;76(12):1125-9.

18. Moseley AM, Yap MC. Interrater reliability of the TEMPA for the measurement of upper limb function in adults with traumatic brain injury. J Head Trauma Rehabil. 2003;18(6):526-31.

19. Nedelec B, Dion K, Correa JA, Desrosiers J. Upper extremity performance test for the elderly (TEMPA): normative data for young adults. J Hand Ther 2011;24(1):31-43.

20. van Lankveld W, van't Pad BP, Bakker J, Terwindt S, Franssen M, van Kiel P. Sequential occupational dexterity assessment (SODA): a new test to measure hand disability. J Hand Ther. 1996;9(1):27-32.

21. van Lankveld WG, Graff MJ, van't Pad Bosch PJ. The short version of the sequential occupational dexterity assessment based on individual tasks' sensitivity to change. Arthritis Care Res. 1999;12(6):417-24.

22. Aaron DH, Jansen CWS. Development of the Functional Dexterity Test (FDT): construction, validity, reliability, and normative data. J Hand Ther. 2003;16(1): 12-21.

23. Sartorio F, Bravini E, Vercelli S, Ferriero G, Plebani G, Foti C, Franchignoni F. The Functional Dexterity Test: Test-retest reliability analysis and up-to date reference norms. J Hand Ther. 2013;26(1):62-8.

24. Gable C, Chau N, Xénard J, André J. Accords inter-juges d'une mesure fonctionnelle analytique du membre supérieur. Revue d'epidemiologie et de sante publique. 1993;41(1):72-83.

25. Luquet C, Chau N, Nadif M, Guillemin F, Gavillot C, Petry D, Moreau T, Bourgkard E, Hecquet B, André J. Unidimensionnalité d'une mesure fonctionnelle pour des malades ayant une lésion du membre supérieur. Revue d'epidemiologie et de sante publique. 1996;44(3):248-61.

26. GABLE C, XENARD J, J-M ANDRE. Bilan 400 points. Journal d'ergothérapie. 1996;18(2):47-58.

27. Gable C,Xenard J, Makiela E, Chau N. Evaluation focntionnelle de la main.Bilan 400 points et tests chiffrés. Ann Readaptation Med Phys. 1997:40:95-101.

28. Greenseid DZ, McCormack RM. Functional hand testing: a profile evaluation. Plast Reconstr Surg. 1968;42(6):567-71.

29. Jacobson-Sollerman C, Sperling L. Grip function of the healthy hand in a standardized hand function test. A study of the Rancho Los Amigos test. Scand J Rehabil Med. 1977;9(3):123-9.

30. Konzelmann M, Gable C, Angel S, Balandras S, Fortel Y, Gauzelin M-P, Gusse I, Monereau C, Coutant M-EM, Sanamane V. Quels sont les items de la Classification internationale du fonctionnement (CIF) couverts par le bilan 400 points? Consensus entre deux équipes de thérapeutes. Ann Physical Rehabil Med. 2012;55:e292.

31. Naranjo Olguín J, Escalona D’Angelo P, Solís Flores F. San Martín Peñailillo P: Pauta funcional de mano Bilan 400 points validada en población de 7 a 17 años de edad portadora de discapacidad neuro-músculo-esquelética. Rehabilitación. 2014;48(3):151-9.

32. Gable C, Kandel M, Moureau F, Beer L, Chau N, Paysant J. Étude de reproductibilité de la cotation du «Bilan 400 points», une mesure de capacité fonctionnelle de la main. Chir Main. 2012;31(2):76-82.
33. Angst F, Drerup S, Werle S, Herren DB, Simmen BR, Goldhahn J. Prediction of grip and key pinch strength in 978 healthy subjects. BMC Musculoskelet Disord. 2010;11(1):94.

34. Anthoine E, Moret L, Regnault A, Sébille V, Hardouin J-B. Sample size used to validate a scale: a review of publications on newly-developed patient reported outcomes measures. Health Qual Life Outcomes. 2014;12(1):2.

35. Mokkink LB, Terwee CB, Knol DL, Stratford PW, Alonso J, Patrick DL, Bouter LM, De Vet HC. The COSMIN checklist for evaluating the methodological quality of studies on measurement properties: a clarification of its content. BMC Med Res Methodol. 2010;10(1):22.

36. Mokkink LB, Terwee CB, Patrick DL, Alonso J, Stratford PW, Knol DL, Bouter LM, de Vet HC. The COSMIN study reached international consensus on taxonomy, terminology, and definitions of measurement properties for health-related patient-reported outcomes. J Clin Epidemiol. 2010;63(7):737-45.

37. Fermanian J. Validation des échelles d'évaluation en médecine physique et de réadaptation: comment apprécier correctement leurs qualités psychométriques. Ann Readapt Med Phys. 2005;48(6):281-7.

38. Bland JM, Altman D. Statistical methods for assessing agreement between two methods of clinical measurement. Lancet. 1986;327(8476):307-10.

39. de Vet HC, Terwee CB, Ostelo RW, Beckerman H, Knol DL, Bouter LM. Minimal changes in health status questionnaires: distinction between minimally detectable change and minimally important change. Health Qual Life Outcomes. 2006;4(1):54.

40. Portney LG, Watkins MP. Foundations of clinical research: applications to practice; 2009

41. Rudman D, Hannah S. An instrument evaluation framework: description and application to assessments of hand function. J Hand Ther. 1998;11(4):266-77.

42. Fayad F, Lefevre-Colau M-M, Gautheron V, Macé Y, Fermanian J, MayouxBenhamou A, Roren A, Rannou F, Roby-Brami A, Revel M. Reliability, validity and responsiveness of the French version of the questionnaire Quick Disability of the Arm, Shoulder and Hand in shoulder disorders. Man Ther. 2009;14(2):206-12.

43. http://www.dash.iwh.on.ca/.

44. Ferreira PL. Development of the Portuguese version of MOS SF-36. Part I. cultural and linguistic adaptation. Acta Med Port. 2000;13(1-2):55-66.

45. Severo $M$, Santos AC, Lopes $C$, Barros $H$. Reliability and validity in measuring physical and mental health construct of the Portuguese version of MOS SF36. Acta Med Port. 2006;19(4):281-7.

46. Leplege A, Mesbah M, Marquis P. Preliminary analysis of the psychometric properties of the French version of an international questionnaire measuring the quality of life: the MOS SF-36 (version 1.1). Revue d'epidemiologie et de sante publique. 1995;43(4):371-9.

47. Decourcelle F, Decourcelle C, Rigaux P. Apport du Jebsen test dans l'évaluation du membre supérieur en neurologie centrale. J d'ergotherapie. 1996;18(2):64-71.

48. Ferreiro KN,dos santos RL, Conforto AB. Psychometric properties of the portuguese version of the jebsen-taylor test for adults with mild hemiparesis. Rev Bras Fisioter. 2010;14(5):377-81.

49. Wright A, Hannon J, Hegedus EJ, Kavchak AE. Clinimetrics corner: a closer look at the minimal clinically important difference (MCID). J Man Manip Ther. 2012;20(3):160-6

50. Youden WJ. Index for rating diagnostic tests. Cancer. 1950;3(1):32-5

51. Revicki D, Hays RD, Cella D, Sloan J. Recommended methods for determining responsiveness and minimally important differences for patient-reported outcomes. J Clin Epidemiol. 2008;61(2):102-9.

52. Terwee CB, Bot SD, de Boer MR, van der Windt DA, Knol DL, Dekker J, Bouter $L M$, de Vet HC. Quality criteria were proposed for measurement properties of health status questionnaires. J Clin Epidemiol. 2007;60(1):34-42.

53. Beaton D, Bombardier C, Katz J, Wright J. A taxonomy for responsiveness. J Clin Epidemiol. 2001;54(12):1204-17.

54. Schmitt JS, Di Fabio RP. Reliable change and minimum important difference (MID) proportions facilitated group responsiveness comparisons using individual threshold criteria. J Clin Epidemiol. 2004;57(10):1008-18.

55. Franchignoni F, Vercelli S, Giordano A, Sartorio F, Bravini E, Ferriero G. Minimal clinically important difference of the disabilities of the arm, shoulder and hand outcome measure (DASH) and its shortened version (QuickDASH). J Orthop Sports Phys Ther. 2014;44(1):30-9.

56. Polson K, Reid D, McNair PJ, Larmer P. Responsiveness, minimal importance difference and minimal detectable change scores of the shortened disability arm shoulder hand (QuickDASH) questionnaire. Man Ther. 2010;15(4):404-7. 
57. Smith-Forbes EV, Howell DM, Willoughby J, Pitts DG, Uhl TL. Specificity of the minimal clinically important difference of the quick disabilities of the arm shoulder and hand (QDASH) for distal upper extremity conditions. J Hand Ther. 2016:29(1):81-8.

58. MacDermid JC, Mulè M. Concurrent validity of the NK hand dexterity test. Physiother Res Int. 2001;6(2):83-93.

59. Chryssochou E, Hilfiker R, Deriaz O, Luthi F, Konzelmann M. La validité de construit convergente (VCC) du bilan 400 points est meilleure avec le sousscore fonction du questionnaire PRWE (patient rated wrist evaluation) version française (F) qu'avec le sore total. Ann Phys Rehabil Med. 2011;54: e64.

60. Kennedy CA, Beaton DE, Smith P, Van Eerd D, Tang K, Inrig T, HoggJohnson S, Linton D, Couban R. Measurement properties of the QuickDASH (disabilities of the arm, shoulder and hand) outcome measure and crosscultural adaptations of the QuickDASH: a systematic review. Qual Life Res. 2013;22(9):2509-47.

61. Copay AG, Subach BR, Glassman SD, Polly DW Jr, Schuler TC. Understanding the minimum clinically important difference: a review of concepts and methods. Spine J. 2007;7(5):541-6.

62. de Vet HC, Ostelo RW, Terwee CB, van der Roer N, Knol DL, Beckerman H, Boers M, Bouter LM. Minimally important change determined by a visual method integrating an anchor-based and a distribution-based approach. Qual Life Res. 2007;16(1):131.

\section{Publisher's Note}

Springer Nature remains neutral with regard to jurisdictional claims in published maps and institutional affiliations.

Ready to submit your research? Choose BMC and benefit from:

- fast, convenient online submission

- thorough peer review by experienced researchers in your field

- rapid publication on acceptance

- support for research data, including large and complex data types

- gold Open Access which fosters wider collaboration and increased citations

- maximum visibility for your research: over $100 \mathrm{M}$ website views per year

At $\mathrm{BMC}$, research is always in progress.

Learn more biomedcentral.com/submissions 